\title{
Effect of Underwater Gait Training with a Progressive Increase in Speed on Balance, Gait, and Endurance in Stroke Patients
}

\author{
Heejoong Kim', Yijung Chung ${ }^{2}$ \\ 'Department of Physical Therapy, The Graduate School, Sahmyook University; ${ }^{2}$ Department of Physical Therapy, College of Health Science and \\ Social Welfare, Sahmyook University, Seoul, Korea
}

Purpose: This study aimed to investigate the effect of progressive speed increase during underwater gait training on stroke patients' balance, gait, and endurance, as well as to compare the effects of underwater gait training and land gait training.

Methods: Subjects were randomly allocated into three groups. Underwater gait training group $(n=10)$, land gait training group $(n=9)$ and control group $(n=9)$. The groups performed their respective programs as well as conventional physical therapy 3 times/week for 8 weeks. The patients were assessed before and after the experiment in terms of the Berg balance scale, characteristics of gait, and 6-minute walking test.

Results: The beneficial effect perceived in the speed increase underwater gait training (UGT) group was significantly greater than in the groups who were trained with speed increase land gait training (LGT) group, and the control group regarding the following aspects: the Berg balance scale, the affected step length, the affected stride length, and the 6-minute walking test $(p<0.05)$. The LGT group showed a more significant effect on the Berg balance scale, the affected step length, the affected stride length, and the 6-minute walking test $(p<0.05)$, compared to the control group. Furthermore, the UGT group showed a significantly greater effect on the gait speed when compared to the control groupb $(\mathrm{p}<0.05)$.

Conclusion: This study shows that progressive UGT is effective in improving balance, gait, and endurance in stroke patients. Therefore, we believe that progressive UGT may be used as a method for general physical therapy in patients with stroke.

Keywords: Locomotion, Gait, Immersion, Stroke

\section{Introduction}

The most common symptom following a stroke is the loss of muscle strength on the affected side. The resulting imbalance of muscle strength leads to asymmetrical posture and inability to support the center of gravity of the trunk on the weight support surface. ${ }^{1}$ As a result of these problems, the patients tend to stand with $61 \%-81 \%$ of the total body weight biased towards the affected limb. ${ }^{2}$ This posture is exacerbated by a compensatory action and affects the body movement, in general. ${ }^{3}$ Weight bias has direct impacts on body movement, e.g. it causes instability in gait, and decreases gait speed. Furthermore, it increases the risk of falls, so that about $25 \%$ of stroke patients experience falls. ${ }^{4}$ Due to the bilateral asymmetry, it is also

Received Jul 1, 2019 Revised Aug 2, 2019

Accepted Aug 27, 2019

Corresponding author Yijung Chung

E-mail yijung36@syu.ac.kr difficult to maintain a normal balance in sitting and standing postures as well as in walking. ${ }^{5}$ This locomotion disorder is accompanied by a decreased endurance or degraded cardiopulmonary function. ${ }^{6}$

Recently, various treatment methods for stroke patients have been reported, among them, underwater exercise promotes diminished burden on the lower extremities joints by using buoyancy and resistance of water, enhancing muscle strength, muscle endurance, balance ability, cardiopulmonary endurance. ${ }^{7}$ Compared to landbased treatments, underwater exercise enables stable training of patients presenting hemiplegia as well as it provides the psychological sense of stability so that the patients are able to exercise with confidence, leading to a greater effect of the exercise. ${ }^{8}$ The physiological

Copylight (C2019 The Korean Society of Physical Therapy

This is an Open Access article distribute under the terms of the Creative Commons Attribution Non-commercial License (Http:// creativecommons.org/license/by-nc/4.0.) which permits unrestricted non-commercial use, distribution, and reproduction in any medium, provided the original work is properly cited. 
responses of the body also include lowering of the heart rate and deepening of the breathing, thereby causing to the natural effect of bradycardia leading to a sense of mental stability. ${ }^{9}$ For these reasons, underwater exercise is often recommended for elderly patients and stroke patients in order to cope with pathological pain in general. ${ }^{710-13}$

In recent years, studies focusing on underwater gait have been conducted and reported results including the improvement in muscle activity, heart rate, maximal oxygen uptake, stance stability, and symmetry through underwater gait. ${ }^{14-22}$ However, in most studies, there were no interventions regarding gait velocity in water. Although Masumoto et al. ${ }^{23}$ recently reported an increase in the rated perceived exertion (RPE) and metabolic consumption as underwater gait speed increases, there was no report on the kinematic characteristics according to the speed. Additionally, most of the studies were conducted in the elderly or normal adults. Therefore, this study aims to investigate the effect of gait training on the balance, gait, and endurance of stroke patients by applying the same intervention regarding speed in exercises performed both in the water and on the ground

\section{Methods}

\section{Participants}

We selected 30 adult stroke patients who were admitted to the Hospital B in Gyeonggi-do, and participated in the rehabilitation program. We randomized hemiplegia patients who were diagnosed with stroke into three groups (Table 1). The inclusion criteria were as follows: 1) patients who were capable of independent gait for more than $10 \mathrm{~m}$ and presented visual and spatial cognitive ability, ${ }^{24}$ 2) patients who were capable of independent gait for more than $15 \mathrm{~min}$ utes, ${ }^{25} 3$ ) subjects who scored 24 or higher in the Korean mini-mental state examination (MMSE-K), 4) patients who did not have pre-

Table 1. Characteristics of the participants in this study

$(n=28)$

\begin{tabular}{lcccc}
\hline Variables & UGT $(n=10)$ & LGT $(n=9)$ & CG $(n=9)$ & $F / \chi^{2}(p)$ \\
\hline Gender & 7 & 7 & 5 & $1.05(0.59)$ \\
Male & 3 & 2 & 4 & \\
Female & $53.1 \pm 15.01$ & $57.22 \pm 11.18$ & $50.33 \pm 19.61$ & $0.44(0.65)$ \\
Age (years) & $168.9 \pm 7.23$ & $164.33 \pm 8.93$ & $169.11 \pm 3.44$ & $1.38(0.27)$ \\
Height (cm) & $68.59 \pm 14.42$ & $62.2 \pm 9.39$ & $62.9 \pm 6.92$ & $1.01(0.38)$ \\
Body weight $(\mathrm{kg})$ & 68.9
\end{tabular}

Mean $\pm S D, U G T$ : speed increase underwater gait training, LGT: speed increase land gait training, CG: control group. vious history or problems of cardiovascular surgery, ${ }^{18}$ and 5) those who gave his/her consent to the participation in the study. Among these patients, those who were afraid of water, who presented uncontrolled seizure experiences, and those who had undergone orthopedic surgery within the last 6 months were excluded from the study. ${ }^{18}$ This study was conducted with those patients and the ones who were accompanied by a caregiver who understood the experiment. The patients signed a written consent form prior to participating in the study following sufficient explanation on the expected effects and side effects. The study was conducted after the approval from the Institutional Review Board (IRB) of Sahmyook University(IRB No. SYUIRB2014-093).

\section{Experiment method}

\section{1) Procedure}

The experiment was conducted for 8 weeks. We assessed balance ability, gait ability, and endurance. The 30 subjects selected were divided into three groups: speed increase underwater gait training (UGT) group, speed increase land gait training (LGT) group, and control group according to the intervention method. All subjects underwent general physical therapy five times a week for $30 \mathrm{~min}$ utes per section. Additionally, the UGT group trained three times a week for 30 minutes per section. The LGT group underwent the intervention three times a week for 30 minutes per section. In the control group, land gait training was conducted three times a week for 30 minutes per section. The training was set to 5 minutes of warm up, 20 minutes of gait training, and 5 minutes of cool down. We performed general physical therapy, which is an exercise-based therapy conducted during the hospitalization and neurodevelopmental treatment. The therapy is based on the traditional motor development theory and on the exercise learning theory. During the training, we experienced one dropout from the LGT group and one from the land gait training group. Therefore, after the training, 10 patients from the UGT group, 9 from the LGT group, and 9 from the control group were measured for their balance ability, spatiotemporal gait ability, and endurance as post-hoc analysis. The subjects of all the three groups participated in the program according to the designated schedule. There were three research assistants for the main research and four research assistants to perform the study measurements. Before conducting the experiments and the measurements, instruction and exercises on how to use the equipment, 
measurement methods, and on the training progress were given in order to minimize errors that could have occurred during the experiment. During gait training, patients were followed from behind to ensure their safety.

\section{(1) speed increase underwater gait training (UGT)}

UGT was conducted in a $4 \times 6$-meter pool at a water temperature of $34^{\circ} \mathrm{C}^{18}$ and a water depth of $1.2 \mathrm{~m}$ for 30 minutes. In the gait training, we used the metronome beat according to the stride length frequency as a means to perform the speed intervention. The metronome was used in a smartphone, and a waterproof smartphone case was used due to the underwater environment. In order to measure the stride length frequency at a comfortable speed in the water, the patient's comfortable speed was measured three times, and the mean value was calculated. For each measurement, gait was conducted for 5 minutes and there was a 1-minute break between every measurement. ${ }^{23}$

In the first week, we measured the patients' stride length frequency by assessing the UGT at a comfortable speed in the water. ${ }^{23}$ During the measurements, we used the metronome beat according to the measured stride length frequency. All the gait training, including straight line motion and rotation, was conducted underwater.

In the third week, gait training was conducted using with the metronome's tempo increased by 3 in relation to the stride length at a comfortable speed, which was measured in the first week, according to the patient's ability. In the fifth week, gait training was conducted using the metronome's tempo increased by 6 when compared to the stride length at a comfortable speed, which was measured in the first week, according to the patient's ability. In the seventh week, gait training was conducted using the metronome's tempo increased by 9 in relation to the stride length at a comfortable speed, which was measured in the first week, according to the patient's ability. In the study of Masumoto et al., ${ }^{23}$ for normal adults, interventions regarding speed should be set with the additional stride length frequency being 5,10, and 15 at preferred stride frequency (PSF). In this study, considering that the subjects are stroke patients, the additional stride length frequency was set as 3, 6, and 9 . All patients were able to gait at the increased rate employed.

\section{(2) speed increase land gait training (LGT)}

The tool used in the LGT was a digital metronome. This training was conducted for 30 minutes in a treatment room with markings sized $4 \times 6 \mathrm{~m}$. In the gait training, we used the metronome beat adjusted according to the stride length frequency at a comfortable speed as a means to perform the speed intervention. For the measurement of PSF, gait was conducted three times and their mean value was obtained so that the patients were able to walk at a comfortable speed. ${ }^{19}$ The speed increase at each period was set to be the same as for the underwater gait training group.

\section{(3) control group}

The control group was placed in the treatment room with the markings measuring $4 \times 6 \mathrm{~m}$ and the land gait training was conducted for 30 minutes. In gait training, speed intervention was performed using the metronome beat adjusted according to the stride length frequency at a comfortable speed. For the measurement of PSF, gait was conducted three times and their mean value was obtained so that the patients were able to walk at a comfortable speed. ${ }^{23}$ We measured the stride length frequency at a comfortable speed on land during the land gait training. ${ }^{23}$ We conducted using the gait training, including straight line motion and rotation, on land, for 8 weeks using the metronome beat adjusted according to the measured gait speed.

\section{2) Tools}

We measured the results using four clinical tests with high reliability, validity, and sensitivity. The Berg Balance Scale was used to assess balance ability, the spatiotemporal gait ability test was used to assess gait ability, and the 6-minute walking test was used to assess endurance. In this study, a single-blind method was used and the assessment, analysis and training were conducted by a therapist with 3 years of clinical experience who was unaware of the group to which each patient belonged.

\section{(1) Berg balance scale (BBS)}

The Berg Balance Scale, a clinical test that measures the sense of balance, consists of 14 items, which may be largely divided into three areas: sitting, standing, and postural changes. The score ranges from the minimum of 0 points to 4 points for each item so that the maximum sum of the 14 items was 56 points. The higher the score assessed, the better the balance was considered. In this test, a score of 45 or less suggests that an assisting device, e.g. a cane, is needed 
during the gait. Scores between 0 and 20 represent a very high risk of falls. Additionally, this tool showed high reliability and internal validity in assessing the balance ability, with $r=0.99$ and $r=0.98$ for the intra-tester reliability and inter-tester reliability, respectively. ${ }^{26}$ Moreover, the internal consistency was 0.97 for the stroke patients, which may be considered as a very valid assessment tool. ${ }^{27}$ In this study, before the measurement, the patient was fully informed of the content by a demonstration and a preliminary exercise.

\section{(2) Spatiotemporal gait ability test}

Spatiotemporal gait ability test measures temporal and spatial gait abilities by using a gait analyzer (GAITRite, CIR system Inc, USA, 2008) to collect quantitative data of the patient's gait types. ${ }^{28}$ The test reliability was $r=0.90 .{ }^{29}$ Additionally, the internal consistency for stroke patients was 0.92 to 0.97 , which shows that this is a very valid assessment tool..$^{30}$ The gait ability test was performed with the subject walking at a comfortable speed, the measurement was taken 3 times and the mean value was used. The shoes and walking aids were used according to the characteristics of the patient and the measurements were made under the same conditions in the preand post-tests. In order to exclude the gait acceleration period and deceleration period, the subjects were allowed to walk at a comfortable gait speed for $2 \mathrm{~m}$ before and including the gait plate. The gait speed and cadence for temporal gait ability, and step length and stride length for spatial gait ability were collected and analyzed.

\section{(3) Six-minutes walking test}

The 6-minute walking test was used to assess endurance. The 6-minute walking test showed high reliability $(\mathrm{ICC}=0.94)$ as a measure of the maximum walkable distance for 6 minutes. ${ }^{31}$ The assessment method required the patients to go back and forth along a gait passage of $25 \mathrm{~m}$, which had markers every $1 \mathrm{~m}$, in its total length without assistance for 6 minutes. The gait speed and break time were adjusted according to the patient's ability.

\section{3) Data Analysis}

The data collected in this study were analyzed using the software SPSS 21.0 for Windows. The statistical significance level $(\alpha)$ was set as 0.05 . The general characteristics of patients were analyzed using descriptive statistics, and normality tests were performed using the Shapiro-Wilk test. The pre-experiment homogeneity test was con- ducted for subjects' general characteristics, and dependent variables were analyzed by chi-square test and one-way ANOVA. We used the paired t-test to respectively verify the difference between preand post-test mean values within the group. One-way ANOVA was performed to compare the differences in variation between the groups. The least squares difference (LSD) method was used for post-hoc analysis to explain differences between the groups after the test.

\section{Results}

\section{Change in the Berg Balance Scale results}

The patients in both the UGT group and in the LGT group improved significantly regarding the Berg balance scale, when comparing the results achieved before and after the intervention in relation to the control group $(\mathrm{p}<0.05)$. The UGT group showed a significantly higher improvement when compared to LGT group regarding the results before and after the intervention (Table 2).

\section{Change in spatiotemporal gait ability}

The UGT group showed a significant improvement (pre- and postintervention difference, hereinafter difference, 4.05) in gait speed before and after the experiment compared to land gait training (difference, 0.62) $(\mathrm{p}<0.05)$. The UGT group (difference 4.72) and the LGT group (difference 2.66) showed significant improvement in affected step length when compared to the control group (difference 0.56) $(\mathrm{p}<0.05)$ and the UGT group showed significant improvement when compared to LGT group $(\mathrm{p}<0.05)$. Additionally, the UGT group (difference 7.07) and the LGT group (difference 3.90) displayed significant improvement in affected stride length when compared to the control group (difference 0.67) $(\mathrm{p}<0.05)$ and UGT group showed significant improvement when compared to LGT group $(\mathrm{p}<0.05)($ Table 2$)$.

\section{Six-minute walking test}

The UGT group (4.05) showed a significant improvement $(\mathrm{p}<0.05)$ in gait speed when compared to land gait training (0.62) and UGT group ( $\mathrm{p}<0.05)$. The LGT group (2.66) displayed a significant increase $(\mathrm{p}<0.05)$ in affected step length compared to the control group (0.56). The underwater gait training group improved significantly $(\mathrm{p}<0.05)$ when comparing the results before and after the in- 
Table 2. Changes in balance, gait, endurance in this study

\begin{tabular}{|c|c|c|c|c|c|}
\hline Item & & $\operatorname{UGT}(\mathrm{A})$ & LGT (B) & CG (C) & $F(p)$ post-hoc analysis \\
\hline \multirow[t]{4}{*}{ BBS } & Before experiment & $46.20 \pm 4.08$ & $46.20 \pm 4.08$ & $45.22 \pm 4.55$ & \\
\hline & After experiment & $52.70 \pm 2.16$ & $48.22 \pm 3.03$ & $46.33 \pm 4.09$ & \\
\hline & Difference & $6.50 \pm 3.89$ & $2.78 \pm 1.72$ & $1.11 \pm 1.05$ & $7.985(0.002)$ \\
\hline & $t(p)$ & $-5.278(0.001)$ & $-4.865(0.001)$ & $-3.162(0.013)$ & $A|B| C$ \\
\hline \multirow[t]{4}{*}{ Gait speed (cm/s) } & Before experiment & $57.22 \pm 41.60$ & $62.24 \pm 34.42$ & $56.16 \pm 18.67$ & \\
\hline & After experiment & $61.27 \pm 42.81$ & $65.05 \pm 35.33$ & $56.78 \pm 19.10$ & \\
\hline & Difference & $4.05 \pm 1.73$ & $2.81 \pm 2.64$ & $0.62 \pm 2.28$ & $5.684(0.009)$ \\
\hline & $t(p)$ & $-7.396(<0.001)$ & $-3.186(0.013)$ & $-.811(0.441)$ & $A \mid C$ \\
\hline \multirow[t]{4}{*}{ Cadence (steps/min) } & Before experiment & $78.45 \pm 19.11$ & $85.83 \pm 18.86$ & $80.28 \pm 16.98$ & \\
\hline & After experiment & $80.80 \pm 18.57$ & $87.39 \pm 17.48$ & $80.86 \pm 16.31$ & \\
\hline & Difference & $2.45 \pm 0.82$ & $2.14 \pm 2.64$ & $0.58 \pm 2.16$ & $2.351(0.116)$ \\
\hline & $t(p)$ & $-9.455(<0.001)$ & $-2.429(<0.001)$ & $-.802(0.446)$ & \\
\hline \multirow[t]{4}{*}{ Affected step length $(\mathrm{cm})$} & Before experiment & $40.16 \pm 17.99$ & $40.05 \pm 15.22$ & $41.76 \pm 9.02$ & \\
\hline & After experiment & $44.88 \pm 17.46$ & $42.71 \pm 14.65$ & $42.32 \pm 7.59$ & \\
\hline & Difference & $4.72 \pm 1.70$ & $2.66 \pm 1.45$ & $0.56 \pm 3.04$ & $8.779(0.001)$ \\
\hline & $t(p)$ & $-8.787(<0.001)$ & $-5.493(0.001)$ & $-0.558(0.592)$ & $A|B| C$ \\
\hline \multirow[t]{4}{*}{ Affected stride length $(\mathrm{cm})$} & Before experiment & $80.28 \pm 35.98$ & $80.36 \pm 29.78$ & $82.92 \pm 17.40$ & \\
\hline & After experiment & $87.35 \pm 35.38$ & $84.27 \pm 28.68$ & $83.59 \pm 14.46$ & \\
\hline & Difference & $7.07 \pm 2.02$ & $3.90 \pm 3.35$ & $0.67 \pm 4.19$ & $9.055(0.001)$ \\
\hline & $t(p)$ & $-11.048(<0.001)$ & $-3.489(0.008)$ & $-.481(0.644)$ & $A|B| C$ \\
\hline \multirow[t]{4}{*}{$6 \mathrm{MWT}(\mathrm{m})$} & Before experiment & $210.70 \pm 76.99$ & $192.51 \pm 80.81$ & $207.11 \pm 71.12$ & \\
\hline & After experiment & $233.08 \pm 75.30$ & $203.99 \pm 85.59$ & $209.15 \pm 65.93$ & \\
\hline & Difference & $22.37 \pm 10.67$ & $11.48 \pm 15.19$ & $2.04 \pm 6.06$ & $7.771(0.002)$ \\
\hline & $t(p)$ & $6.633(<0.001)$ & $2.266(0.53)$ & $1.010(0.342)$ & $A|B| C$ \\
\hline
\end{tabular}

Mean \pm SD, UGT: speed increase underwater gait training, LGT: speed increase land gait training, CG: control group, BBS: berg balance scale, 6MWT: 6-minute walk test

tervention when compared to the LGT group. Furthermore, the UGT group (pre- and post-treatment, 7.07) and the LGT group (preand post-intervention 3.90) showed significant improvement in affected stride length when compared to the control group (0.67) $(\mathrm{p}<0.05)$. The UGT group improved significantly $(\mathrm{p}<0.05)$ regarding the results before and after the intervention when compared to the LGT group (Table 2).

\section{Discussion}

The purpose of this study was to assess the improvement in balance, gait, and endurance by using progressive UGT. Among the underwater exercises, underwater gait has been suggested as a method to improve the function of the lower limbs. Its therapeutic advantages are largely classified into two groups. First, the weight supported by the lower limb may be reduced by the buoyancy when compared to the gait on land, and, second, the faster is the patient's movement, the more resistance is produced. Exercising in the water not only provides adequate exercise conditions for patients who are unable to bear the weight load due to damage to the lower limbs but also provides an environment in which patients who are unable to exercise on the ground are able to start exercising sooner. ${ }^{18}$

\section{Change in balance ability}

The Berg Balance Scale score of the UGT group patients increased from 45.44 points, before the experiment, to 52.70 points, after the experiment, yielding a difference of 6.50 points, which was statistically significant when compared to other two groups $(\mathrm{p}<0.05)$. In the post-hoc analysis, the UGT group showed a more significant increase than the LGT group and the control group $(\mathrm{p}<0.05)$. Noh et al. ${ }^{32}$ reported that the underwater exercise program for stroke patients showed a significant increase in the Berg balance test as compared to the general physical therapy. Masumoto et al. ${ }^{33}$ found that when the underwater gait was compared with the land gait, higher muscle activity in the lower limbs was observed and that the increased stability of the lower limbs affects the improvement of bal- 
ance ability. ${ }^{34}$ Furthermore, the reason for the improved balance in the water is that if the speed of movement in the water is increased, greater resistance is generated, resulting in the increase in the intensity of the exercise. Moreover, the vortex effect in the water allows the human body to master balance and coordination actions. The results of this study corroborate with these previous studies and it is thought that it might work as factors which may positively affect the improvement of the participants' balance ability.

\section{Changes in gait ability}

The affected step length of the patients in the UGT group increased by $4.72 \mathrm{~cm}$, from $40.16 \mathrm{~cm}$ before the experiment to $44.38 \mathrm{~cm}$ after it and showed a statistically significant increase when compared to the other two groups $(\mathrm{p}<0.05)$. As a result of the post-hoc analysis, speed increase underwater gait group showed a more significant increase when compared to the other two groups $(\mathrm{p}<0.05)$.

Also, in the affected stride length of the UGT group, there was a statistically significant increase when compared to the other two groups, the increase was of $7.07 \mathrm{~cm}$, from $80.28 \mathrm{~cm}$ before the experiment to $87.35 \mathrm{~cm}$ after it $(\mathrm{p}<0.05)$. The UGT group showed a more significant increase than the other two groups in the post-hoc analysis $(\mathrm{p}<0.05)$. Miyoshi et al..$^{35}$ reported that a higher load was applied to the hip joint due to the increased water resistance when fast gait was performed in the water. In the study of Barela et al., ${ }^{14}$ as a result of analyzing the effects of land gait and underwater treadmill gait, the activities of tensor fasciae latae, vastus lateralis, and biceps femoris increased in the stance phase in the underwater environment. Moreover, Kaneda et al. ${ }^{36}$ reported that when underwater gait training and land gait training were conducted for adults in general, the maximum contraction force of the rectus femoris was higher in the water than on the ground. This was reported to be due to the fact that the lower limbs require higher force by water resistance. In this study, the authors considered that UGT strengthens the flexor and extensor muscles of the hip joints of the existing side, increasing the stability in posture and the stability in gait stance phase, resulting in an augmented step length and stride length.

\section{Changes in endurance}

The 6-minute walking test of the patients in the UGT group showed a statistically significant increase when compared to the other two groups with an increase of $22.37 \mathrm{~m}$, from $210.70 \mathrm{~m}$ before the ex- periment to $233.08 \mathrm{~m}$ after it $(\mathrm{p}<0.05)$. The post-hoc analysis revealed that the UGT group showed a more significant increase than the other two groups $(\mathrm{p}<0.05)$. Shono et al. ${ }^{37}$ investigated heart rate, maximal oxygen uptake, gait, and lower limb muscle activity while walking on a treadmill on the land and underwater in elderly women. The results showed significantly increased heart rate, decreased cadence, and significantly increased lower extremity muscle activity in the underwater gait during gait at the same speed. Additionally, Chu et al..$^{38}$ reported that the underwater environment increased cardiopulmonary function and maximal oxygen uptake, leading to an increase in gait endurance. The density of water is about 800 times higher than the density of air, the human body is under pressure in the water, and the movement in water is an important factor to increase the energy consumption when compared to the movement in the ground. ${ }^{39}$ Masumoto et $\mathrm{al}^{40}{ }^{40}$ reported higher RPE in the water when compared to the value obtained on the ground This was associated with higher blood lactate concentration, oxygen uptake, heart rate, and lower limb muscle activity in the water. Also, when gait with different stride length cycle was conducted underwater and on land, much higher metabolic consumption and RPE was produced when performing the underwater gait under high stride length cycle conditions when compared to lower stride length cycle conditions. This result was reported to arise from the greater resistance and turbulent flow in the underwater environment in comparison to what happens on the land. ${ }^{40}$ In this study, underwater gait training with fast speed is applied, which is thought to induce increased metabolic consumption by generating large water resistance. Therefore, in line with the purpose of this study, progressive UGT had a positive impact on the improvement of gait endurance in stroke patients.

There are some limitations to this study. The first one is that gait irregularities may have occurred due to the fact that we included rotation gait as well as the straight gait. Second, it is thought that gait speed was influenced by the inability to control the flotation phenomenon in the lower limb of the affected side. This study shows that UGT is effective in improving the balance, gait, and endurance of stroke patients. As mentioned above, UGT was confirmed that training in the water was effective as resistance exercise of the lower limb. Therefore, it is thought that progressive UGT may be used in clinical practice as a training method for general physical therapy in patients with decreased balance, gait, and endurance. 


\section{REFERENCES}

1. Ikai T, Kamikubo T, Takehara I et al. Dynamic postural control in patients with hemiparesis. Am J Phys Med Rehabil. 2003;82(6):463-9.

2. Sackley C, Baguley B. Visual feedback after stroke with the balance performance monitor: two single-case studies. Clinical rehabilitation. 1993; 7(3):189-95.

3. Hocherman S, Dickstein R, Pillar T. Platform training and postural stability in hemiplegia. Arch Phys Med Rehabil. 1984;65(10):588-92.

4. Geiger RA, Allen JB, O'Keefe J et al. Balance and mobility following stroke: Effects of physical therapy interventions with and without biofeedback/forceplate training. Phys Ther. 2001;81(4):995-1005.

5. Eggers O. Terapia ocupacional no tratamento da hemiplegia do adulto. Rio de Janeiro: Colina. 1984:172-88.

6. Dean E, Frownfelter DL. Cardiovascular and pulmonary physical therapy: Evidence and practice. ,St. Louis, Mosby, 2006

7. Bates A, Hanson N. The principles and properties of water. Aquatic exercise therapy. Philadelphia: WB Saunders. 1996:1-320.

8. Matsumoto I, Araki H, Tsuda K et al. Effects of swimming training on aerobic capacity and exercise induced bronchoconstriction in children with bronchial asthma. Thorax. 1999;54(3):196-201.

9. Echt M, Duweling J, Gauer OH et al. Effective compliance of the total vascular bed and the intrathoracic compartment derived from changes in central venous pressure induced by volume changes in man. Circulation research. 1974;34(1):61-8.

10. Devereux K, Robertson D, Briffa NK. Effects of a water-based program on women 65 years and over: A randomised controlled trial. Aust J Physiother. 2005;51(2):102-8.

11. Simmons V, Hansen PD. Effectiveness of water exercise on postural mobility in the well elderly: An experimental study on balance enhancement. J Gerontol A Biol Sci Med Sci. 1996;51(5):M233-8.

12. Suomi R, Collier D. Effects of arthritis exercise programs on functional fitness and perceived activities of daily living measures in older adults with arthritis. Arch Phys Med Rehabil. 2003;84(11):1589-94.

13. Kim H, Chung Y. Comparison of characteristics during backward walking according to various stride frequencies in underwater and ground environments. Physical Therapy Rehabilitation Science. 2018;7(2):83-7.

14. Barela AM, Stolf SF, Duarte M. Biomechanical characteristics of adults walking in shallow water and on land. J Electromyogr Kinesiol. 2006; 16(3):250-6.

15. Hall J, Macdonald I, Maddison P et al. Cardiorespiratory responses to underwater treadmill walking in healthy females. Eur J Appl Physiol Occup Physiol. 1998;77(3):278-84.

16. Masumoto K, Takasugi S-i, Hotta N et al. Electromyographic analysis of walking in water in healthy humans. J Physiol Anthropol Appl Human Sci, 2004;23(4):119-27.

17. Masumoto K, Takasugi S, Hotta $\mathrm{N}$ et al. A comparison of muscle activity and heart rate response during backward and forward walking on an underwater treadmill. Gait Posture. 2007;25(2):222-8.

18. Jung T, Lee D, Charalambous $\mathrm{C}$ et al. The influence of applying additional weight to the affected leg on gait patterns during aquatic treadmill walking in people poststroke. Arch Phys Med Rehabil. 2010;91(1):12936.

19. Park J-H, Chung Y. A comparison of underwater gait training with addi- tional weight application and over-ground gait training to improve balance and lower extremity strength in persons with stroke. The Journal of Korean Physical Therapy. 2017;29(2):101-7.

20. Kum DM, Shin WS. Effect of backward walking training using an underwater treadmill on muscle strength, proprioception and gait ability in persons with stroke. Physical Therapy Rehabilitation Science. 2017;6(3): 120-6.

21. Lim HS, Lee SM. The effect of a water exercise on gait characteristics in the elderly post stroke patients. Physical Therapy Rehabilitation Science. 2012;1(1):17-21.

22. Park SK, Yang DJ, Kang JI et al. The effects of water-based exercise on muscle activity and gait ability in stroke patients. J Kor Phys Ther. 2015; 27(5):369-74

23. Masumoto K, Nishizaki Y, Hamada A. Effect of stride frequency on metabolic costs and rating of perceived exertion during walking in water. Gait Posture. 2013;38(2):335-9.

24. Regnaux JP, Pradon D, Roche N et al. Effects of loading the unaffected limb for one session of locomotor training on laboratory measures of gait in stroke. Clin Biomech (Bristol, Avon). 2008;23(6):762-8.

25. Kang HK, Kim Y, Chung Y et al. Effects of treadmill training with optic flow on balance and gait in individuals following stroke: Randomized controlled trials. Clin Rehabil. 2012;26(3):246-55.

26. Berg K, Wood-Dauphinee S, Williams J. The balance scale: Reliability assessment with elderly residents and patients with an acute stroke. Scand J Rehabil Med. 1995;27(1):27-36.

27. Blum L, Korner-Bitensky N. Usefulness of the berg balance scale in stroke rehabilitation: A systematic review. Phys Ther. 2008;88(5):559-66.

28. van Uden CJ, Besser MP. Test-retest reliability of temporal and spatial gait characteristics measured with an instrumented walkway system (gaitrite). BMC Musculoskelet Disord. 2004;5:13.

29. Bilney B, Morris M, Webster K. Concurrent related validity of the gaitrite walkway system for quantification of the spatial and temporal parameters of gait. Gait \& posture. 2003;17(1):68-74.

30. Hill KD, Goldie PA, Baker PA et al. Retest reliability of the temporal and distance characteristics of hemiplegic gait using a footswitch system. Arch Phys Med Rehabil. 1994;75(5):577-83.

31. Steffen TM, Hacker TA, Mollinger L. Age- and gender-related test performance in community-dwelling elderly people: Six-minute walk test, berg balance scale, timed up \& go test, and gait speeds. Phys Ther. 2002;82(2):128-37.

32. Noh DK, Lim JY, Shin HI et al. The effect of aquatic therapy on postural balance and muscle strength in stroke survivors-a randomized controlled pilot trial. Clin Rehabil. 2008;22(10-11):966-76.

33. Masumoto K, Shono T, Takasugi S et al. Age-related differences in muscle activity, stride frequency and heart rate response during walking in water. J Electromyogr Kinesiol. 2007;17(5):596-604.

34. Suputtitada A, Suwanwela NC, Tumvitee S. Effectiveness of constraintinduced movement therapy in chronic stroke patients. J Med Assoc Thai. 2004;87(12):1482-90.

35. Miyoshi T, Shirota T, Yamamoto $S$ et al. Functional roles of lower-limb joint moments while walking in water. Clin Biomech (Bristol, Avon). 2005;20(2):194-201.

36. Kaneda K, Wakabayashi H, Sato D et al. Lower extremity muscle activity during deep-water running on self-determined pace. J Electromyogr Ki- 
nesiol. 2008;18(6):965-72.

37. Shono T, Masumoto K, Fujishima K et al. Gait patterns and muscle activity in the lower extremities of elderly women during underwater treadmill walking against water flow. J Physiol Anthropol. 2007;26(6):579-86.

38. Chu KS, Eng JJ, Dawson AS et al. Water-based exercise for cardiovascular fitness in people with chronic stroke: A randomized controlled trial. Arch Phys Med Rehabil. 2004;85(6):870-4.
39. di Prampero PE. The energy cost of human locomotion on land and in water. Int J Sports Med. 1986;7(2):55-72.

40. Masumoto K, Shono T, Hotta N et al. Muscle activation, cardiorespiratory response, and rating of perceived exertion in older subjects while walking in water and on dry land. J Electromyogr Kinesiol. 2008;18(4): 581-90. 